\title{
Management of Mishandled Cases of Post-partum Vagino-cervical Prolapse by Recurrence Prevention with Foreign Body in Two Cows
}

\author{
V. Varudharajan*, M. Selvaraju, S. Prakash and K. Ravikumar \\ Department of Veterinary Gynaecology and Obstetrics, Veterinary College and Research \\ Institute, TANUVAS, Namakkal - 637001, Tamil Nadu, India \\ *Corresponding author
}

A B S T R A C T

Keywords

Cervico-vaginal prolapse, Cow, foreign body, Postpartum, Recurrence prevention

Article Info

Accepted: 04 April 2019

Available Online: 10 May 2019

\section{Introduction}

Cervico-vaginal prolapse usually involve protrusion of the portion of the floor, lateral walls and roof of vagina through vulva along with the cervix and uterus, moving caudally (Roberts 1971). Although it may be of multiple etiologies but placental estrogen production during second half of gestation in cattle causing relaxation of pelvic ligament, vulva and vulval sphincter muscle are most feasible proposition although hereditary predisposition may not be undermined (Purohit, 2012). Pessaries consist of a long narrow wine bottle or similar blunt round object inserted into the vagina after replacement (Roberts 1971). The present study describes the management of mishandled cases of post-partum vaginocervical prolapse in two dairy cattle by quacks using foreign bodies, one with glass bottle and another with a stainless steel vessel as a recurrence prevention strategy without proper repositioning.

\section{Case history and clinical observations}

Two Jersey cross bred cows one on its first parity (Case 1) and other on its second parity (Case 2) were brought to the Veterinary 
Clinical Complex (VCC), Veterinary College and Research Institute (VCRI), Namakkal with the history of both the animals calved recently, later showed continuous straining and prolapse of the vagina and cervix. Both the cases were treated by the local quacks, where they failed to reposition the mass to their normal position and prevented the recurrence by suturing the foreign body with the vulva. But due to the irregular reposition, both the animals exhibited continuous straining and brought to VCC, VCRI.

The clinical examination revealed both the animals were anorectic with all other vital parameters in the normal range. By opening the suture made on the vulva, a glass bottle (Fig. 3) tied on its neck attached to the vulval retention suture in one cow and in other a stainless steel vessel (Fig. 4). Both the sutures were made in the vulva not in the hair-line (Fig. $1 \& 2$ ) which caused irritation in turn continuous straining by the animals.

\section{Clinical management}

For both the animals, caudal epidural anaesthesia was achieved by injecting $5 \mathrm{ml}$ of $2 \%$ Lignocaine hydrochloride into sacrococcygeal space. Before correction, the surrounding area of prolapsed masses was washed with soap and prolapsed masses was washed and cleaned with potassium permanganate solution (2\%). Urine was relieved using urinary catheter.

The prolapsed mass was lubricated with Cetrimide cream and repositioned. Further, due to the foreign body, lot of soil and dirt were found in the vagina, which was cleared using Intra-vaginal douche with $2 \% \mathrm{KMnO} 4$ solution. The recurrence prevention was done by vulval retention suture using cross mattress suture pattern in the hair-line. Lacerative wound on the vulva (Fig. 5 \& 6) was cleaned, dressed and Oint. Loraxene applied topically.
The cows were treated with Inj. Ceftriaxone @ $15 \mathrm{mg} / \mathrm{kg}$ i/v for five days, Inj. Dextrose $25 \%$ two liters $\mathrm{i} / \mathrm{v}$ on the first day, Meloxicam @ $0.2 \mathrm{mg} / \mathrm{kg} \mathrm{i} / \mathrm{m}$ for two days, Inj. Calcium Borogluconate $450 \mathrm{ml} \mathrm{i} / \mathrm{v}$ on first day, and Inj. Chlorpheniramine maleate @ $0.5 \mathrm{mg} / \mathrm{kg}$ $\mathrm{i} / \mathrm{m}$ for three days.

Careful observation for 5 days confirming non recurrence of prolapsed, sutures were removed and both the animals recovered uneventfully.

\section{Results and Discussion}

The hormonal alterations or changes taking place at last trimester of pregnancy is believed to be primary cause for prolapse especially estrogen that cause for prolapse especially estrogen that causes relaxation of pelvic ligaments and surrounding soft structures (Wolfe, 2009).

Cervico-vaginal prolapse is a hereditary trait and due to nutritional imbalance contributing to prevalence of vaginal prolapse (Margaux, 2011). More specifically poor quality forage, high level of concentrate, high estrogenic content feeds and hypocalcemia have all been connected with pathology (Mienser and Anderson, 2008). Vaginal contusion at parturition, followed by Fusobacterium necrophorum infection exerts a high degree of irritation with frequent expulsive efforts (Arthur 2001). Lack of myometrial tone and increasedintra-abdominal pressure may also lead to cervico-vaginal prolapse (Kapadiya $e t$ al., 2015). In the reported two cases, the prolapse may be due to increased intraabdominal pressure and improper feeding management. The cases were mishandled by the local quacks which further complicated the condition. Prompt and early corrective treatment by a veterinarian is suggested for this type of cases. 


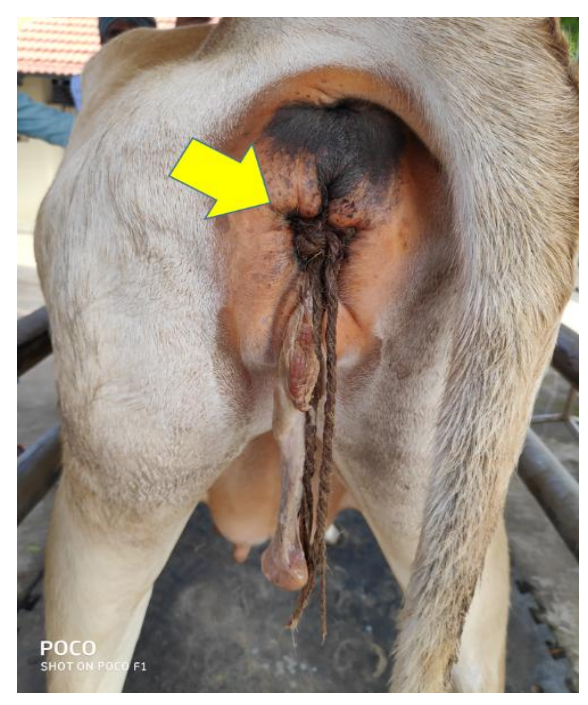

Fig.1 Vulval retention suture on the vulva (Case 1)

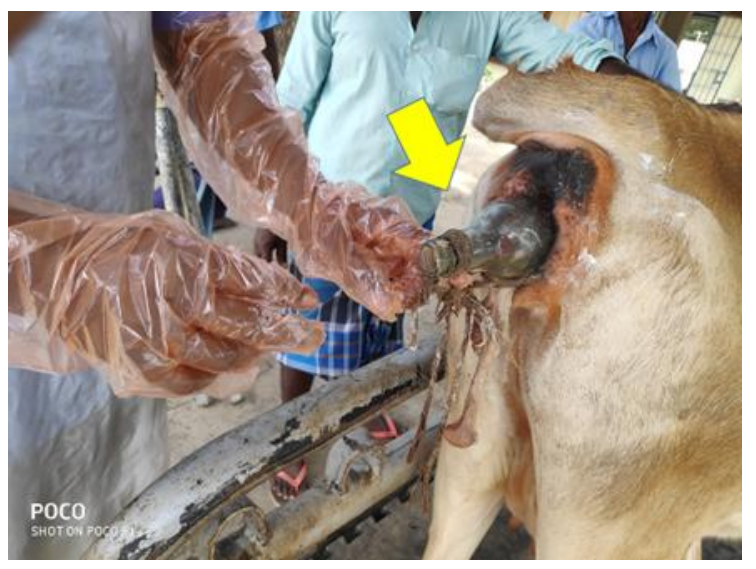

Fig.3 A glass bottle used as a pessary to prevent the recurrence (Case 1)

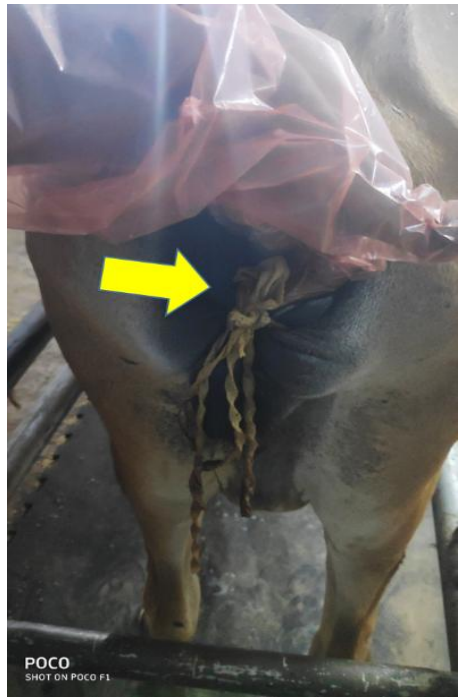

Fig.2 Vulval retention suture on the vulva (Case 2)

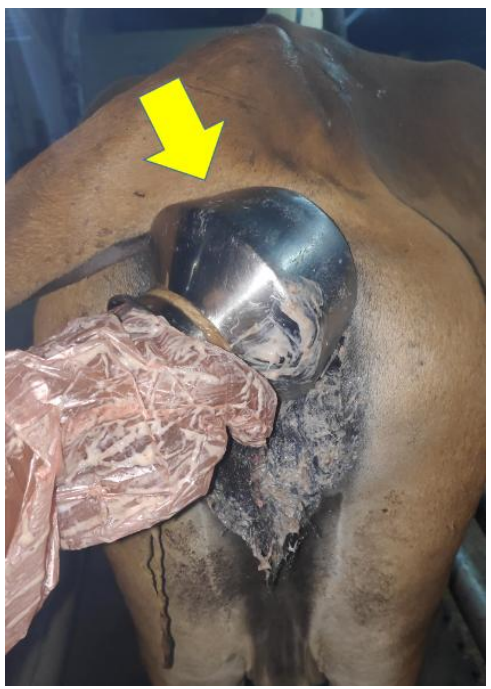

Fig.4 A stainless steel vessel used as a pessary to prevent the recurrence (Case 2) 


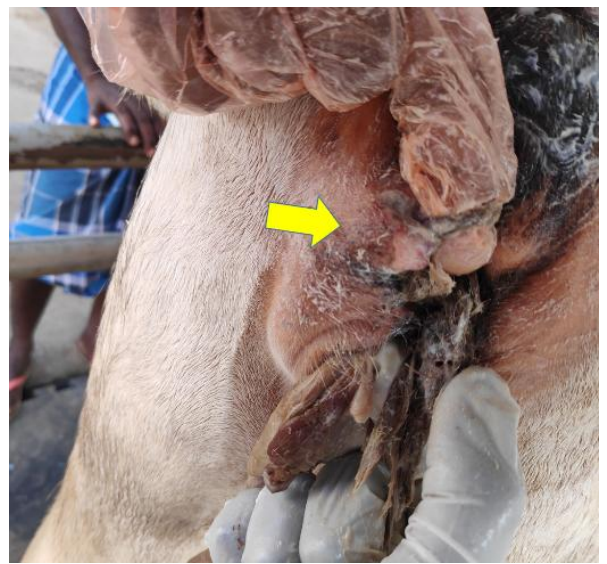

Fig.5 Laceration on the vulva (Case 1)

In the present study, it is concluded that the successful management of mishandled cases of Post-partum vagino-cervical prolapse by quacks using foreign body as recurrence prevention in two cows was recorded.

\section{References}

Arthur, G.H. 2001. Arthur's Veterinary Reproduction and obstetrics, 8th edn. Edited by Noakes DE, Parkinson TJ and Gary CW, Elsevier's, London.

Kapadiya, P.S., Chauhan, P.M., Nakhashi, H.C., Sharma, V.K., Sutaria, T.V. 2015. Recurrent post-partum uterine prolapse in a primiparous Mehsana buffalo- A case report. J Livestock Sci6: 109-112.

Marguax, K. 2011. Study on occurrence of vaginal prolapse in sheep and cattle report submitted to Faculty of Veterinary Medicine Ghent University.

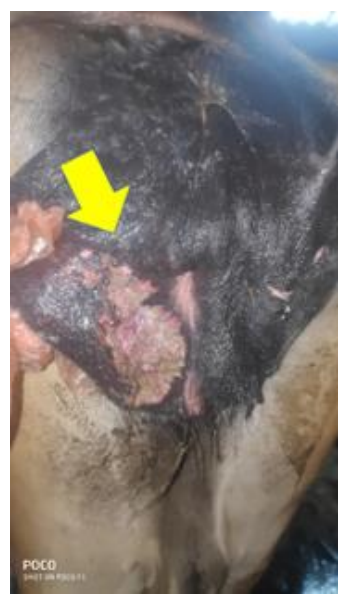

Fig.6 Extensive laceration on the vulva

(Case 2)

Int.J.Curr.Microbiol.App.Sci

(2018) Special Issue-7: 1244-1247.

Mienser, M.D. and Anderson, D.E. 2008. Management of uterine and vaginal prolapse in the bovine. Vet. Clin. Food Anim. Pract., 24: 409-419.

Purohit, G.N.2012. "Etiology, prepartal diagnosis and management of maternal complications of gestation in the water buffalo", Int. J. Appl. Anim. Sci., 1(2): $39-47$.

Robert, S.J. 1971. Veterinary Obstetrics and Genital Diseases (Theriogenology). 2nd ed. (Indian reprint) CBS Publishers and Distributors, New Delhi, India, pp 189196.

Wolfe, D.F. 2009. Medical \& Surgical Management of Vaginal Prolapse in Cattle. Presentation 81st western veterinary conference, Auburn, February 15th, 2009.

\section{How to cite this article:}

Varudharajan, V., M. Selvaraju, S. Prakash and Ravikumar, K. 2019. Management of Mishandled Cases of Post-partum Vagino-cervical Prolapse by Recurrence Prevention with Foreign Body in Two Cows. Int.J.Curr.Microbiol.App.Sci. 8(05): 68-71. doi: https://doi.org/10.20546/ijcmas.2019.805.009 